\title{
VLBA Studies of the Accretion Region in NGC 1275
}

\author{
R. C. Walker ${ }^{1}$, K. I. Kellermann ${ }^{2}$, V. Dhawan ${ }^{1}$, J. D. Romney ${ }^{1}$, K. R. \\ Anantharamaiah $^{3}$ R. C. Vermeulen ${ }^{4}, \&$ W. Alef $^{5}$
}

\begin{abstract}
VLBA observations of the two-sided emission structures in $3 \mathrm{C} 84$ have been used to study the ionized gas in the vicinity of the presumed accretion disk on parsec scales. Strong free-free absorption is seen with a radial gradient, but only upper limits have been obtained on a stimulated recombination line at $23 \mathrm{GHz}$.
\end{abstract}

The parsec-scale structure of the 3C 84 , in the nucleus of NGC 1275 , has a two sided morphology. A simple beaming model, with a mildly relativistic source oriented at $30^{\circ}$ to $50^{\circ}$ to the line-of-sight, fits the relative lengths and brightness of the structures on the two sides and the measured apparent speeds of the structures. With this geometry, properties of the parsec scale accretion region can be studied by comparing the two sides and assuming any differences are due to propagation of the far-side radiation through that region.

Indeed, images made over a range of radio frequencies show that there is strong free-free absorption of radiation from the far-side structure, but not from the near side stucture (Walker et. al. 2000 and references therein). This is strong evidence for the presence of ionized gas in the accretion region, probably associated with the accretion disk. Simple disk theories do not call for high enough temperatures on parsec scales for the gas to be ionized. A likely reason for the presence of such ionized gas is that hard radiation from the immediate vicinity of the core is managing to illuminate the disk because of a warped or flared disk structure, scattering, or because there is gas above the disk in a corona or wind (Levinson, Laor, \& Vermeulen 1995).

In 1995, 3C 84 was observed in January and October with the VLBA at many frequencies including $5.0,8.4,15$, and $22 \mathrm{GHz}$ within a period of about 2 weeks (Walker et. al 2000). Fits for the magnitude and distribution of the absorption show that the spectra are explained well by free-free absorption and not some other mechanism (spectral indices up to 4) and that the absorbing material is distributed with a gradient that decreases with distance from the core. The magnitude of the absorption is consistent with an emission measure that drops from about $10^{9} \mathrm{pc} \mathrm{cm}^{-6}$ at about $1.7 \mathrm{pc}$ from the core to about $10^{8}$ $\mathrm{pc} \mathrm{cm}^{-6}$ at about $3 \mathrm{pc}$. These values assume the the temperature is $10^{4} \mathrm{~K}$ and would need to be modified somewhat for other temperatures. Figure 1 shows images of the 3C 84 system at 22 and $8.4 \mathrm{GHz}$, along with the 2D fit results for the absorption, showing the gradient. Very preliminary analysis of similar

\footnotetext{
${ }^{1}$ National Radio Astronomy Observatory, Socorro, NM U.S.A. ${ }^{2}$ National Radio Astronomy Observatory, Charlottesville, VA U.S.A. ${ }^{3}$ Raman Research Instutute, Bangalore, India. ${ }^{4}$ Netherlands Foundation for Radio Astronomy, Dwingeloo, The Netherlands. ${ }^{5}$ MaxPlank-Institut für Radioastronomie, Bonn, Germany.
} 

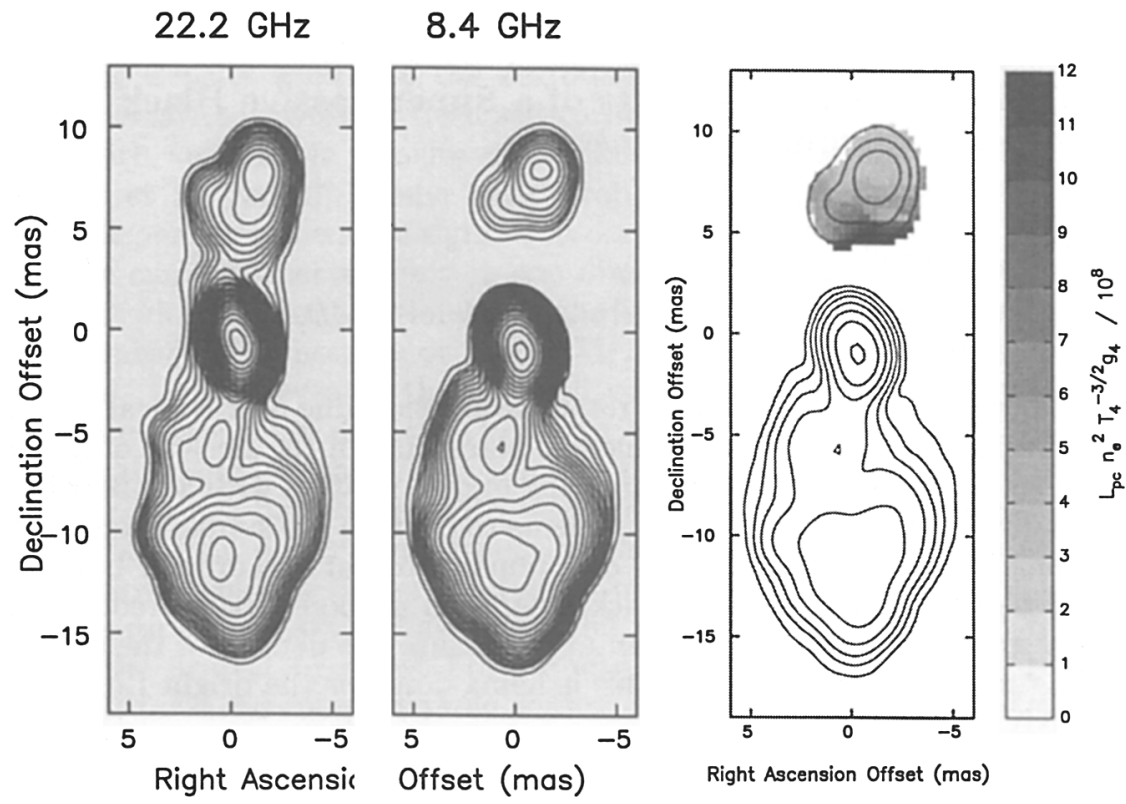

Figure 1. The left two images of 3C 84 are from Oct. 1995 VLBA observations at 22 and $8.4 \mathrm{GHz}$. The contour levels start with 5,10,14 $\ldots \mathrm{mJy}$ beam $^{-1}$ increasing by $\sqrt{2}$. The beam is $1.6 \times 1.2$ mas in PA $0^{\circ}$. The right image shows, in grey scale, the results of a fit of a fit free-free absorption spectrum to $22,15,8.4$ and $5 \mathrm{GHz}$ image intensities. For a fixed temperature of $10^{4} \mathrm{~K}$, the fitted parameter is just the emission measure.

observations made in January 2000 indicate that the source has expanded as expected but that the absorption has not changed significantly.

Under a wide range of physical conditions, but not all, the absorbing gas would be expected to produce stimulated recombination lines. A search was made for the $\mathrm{H} 65 \alpha$ line at $23 \mathrm{GHz}$ over $1486 \mathrm{~km} \mathrm{~s}^{-1}$ in September $1998 \mathrm{using}$ the VLBA and one VLA antenna. No line was detected above the level of about $15 \%$ of the continuum. When combined with the theoretical predictions of line strength under various conditions, this suggests that either the ionized gas is in a thin, dense structure (like a thin disk) or has either a high velocity width or high velocity offset (such as a wind).

The National Radio Astronomy Observatory is a facility of the National Science Foundation, operated under a cooperative agreement by Associated Universities, Inc.

\section{References}

Levinson, A., Laor, A., \& Vermeulen, R. C. 1995, ApJ, 448, 589.

Walker, R. C., Dhawan, V., Romney, J. D., Kellermann, K. I., and Vermeulen, R. C. 2000, ApJ, 530, 233. 